\title{
Failure of Warfarin Anticoagulation Therapy after Administration of Oral Terbinafine
}

\author{
Ivana Štětkářováa,*, Jitka Čupáková
}

\begin{abstract}
Warfarin is widely used anticoagulant drug for a variety of diseases (thromboembolic disease, atrial fibrillation, etc.). It has three most important parallel metabolic pathways, CYP1A2, CYP3A4 and CYP2C9. Terbinafine is a potent CYP2D6 inhibitor. A possible drug interaction could lead to an increased pharmacological effect of the above drugs. Enzyme induction with CYP3A4, CYP2C9, CYP1A2 inducers may have occurred.

Case report: We present a case report of an 88-year-old male patient who has been successfully anticoagulated with warfarin due to atrial fibrillation. He was orally administered terbinafine to treat onychomycosis. Two weeks after initiation of this drug the patient experienced dizziness and feelings of instability, for which he was admitted to the neurology department. A low-efficient INR level was found at the baseline, presumably due to warfarin interaction with terbinafine. The induction of liver enzymes lasts 10-14 days, which matches the introduction of the antifungal agent.

Conclusion: Combined therapy with warfarin and oral terbinafine is actually rarely prescribed but, if used, their interaction can have serious consequences in many clinical situations for which anticoagulation therapy with warfarin is indicated.
\end{abstract}

\section{KEYWORDS}

warfarin; terbinafine; anticoagulation; atrial fibrillation; liver inductor

\section{AUTHOR AFFILIATIONS}

${ }^{1}$ Department of Neurology, Third Faculty of Medicine, Charles University and Faculty Hospital Královské Vinohrady, Prague, Czech Republic

2 Pharmacy Department, Faculty Hospital Královské Vinohrady, Prague, Czech Republic

* Corresponding author: Department of Neurology, Third Faculty of Medicine, Charles University and Faculty Hospital Královské Vinohrady, Ruská 87, Prague 10 - 100 00, Czech Republic; e-mail: ivana.stetkarova@fnkv.cz

Published online: 30 July 2021

Acta Medica (Hradec Králové) 2021; 64(2): 132-135

https://doi.org/10.14712/18059694.2021.23

(c) 2021 The Authors. This is an open-access article distributed under the terms of the Creative Commons Attribution License (http://creativecommons.org/licenses/by/4.0), which permits unrestricted use, distribution, and reproduction in any medium, provided the original author and source are credited. 


\section{INTRODUCTION}

A large number of patients are currently treated with anticoagulants for a variety of diseases (thromboembolic disease, atrial fibrillation, mechanical heart valve, etc.) (1) to prevent ischemic stroke (2). One of the widely used drugs is warfarin, although it is today frequently replaced with direct oral anticoagulants (DOACs) with fewer adverse effects/drug interactions for example in atrial fibrillation (3).

We present a case report of a patient who has been successfully anticoagulated with warfarin due to atrial fibrillation. He was orally administered terbinafine to treat onychomycosis. Two weeks after initiation of this drug the patient experienced dizziness and feelings of instability, for which he was admitted to the neurology department. A low-efficient INR level was found at the baseline, presumably due to warfarin interaction with terbinafine.

\section{CASE REPORT}

This 88-year-old male patient was treated for coronary artery disease with paroxysmal atrial fibrillation, which was managed with warfarin anticoagulation. He also had a history of anterolateral myocardial infarction and a left anterior descending artery (LAD) stent. He had been treated for hyperlipidaemia, hyperuricaemia, impaired glucose tolerance and prostate hypertrophy. He had a history of cholecystectomy and inguinal herniotomy. He used the following oral medication which is summarized in Table 1.

Tab. 1 Patient medication at admission

\begin{tabular}{|l|c|}
\hline \multicolumn{1}{|c|}{ Tramadol/paracetamol } & $\mathbf{7 5} \mathbf{~ m g / 6 5 0 ~} \mathbf{~ m g}$ bid \\
\hline Ezetimib & $10 \mathrm{mg}$ tbl daily \\
\hline Loperamide hydrochloride & $2 \mathrm{mg}$ daily \\
\hline Lansoprasole & $30 \mathrm{mg}$ daily \\
\hline Allopurinol & $100 \mathrm{mg}$ bid \\
\hline Nebivolol & $2.5 \mathrm{mg}$ daily \\
\hline Alprazolam & $0.5 \mathrm{mg}$ daily \\
\hline Trimetazidine & $35 \mathrm{mg}$ bid \\
\hline Propafenone & $15 \mathrm{mg}$ daily \\
\hline Terbinafine & $250 \mathrm{mg}$ daily \\
\hline Warfarin & $5 \mathrm{mg}$ daily \\
\hline
\end{tabular}

The patient arrived at the emergency neurological department due to sudden onset of rotational vertigo which developed during the night. When he sat up in order to go to the toilet, everything began spinning around him. The problems have since continued; he spent the night in bed. The patient perceives no weakness of limbs. He complains of arduous articulation.

In the objective neurological status, the patient was oriented, without aphasia, with mild dysarthria. The blood pressure was $170 / 93$. No nystagmus was observed, only the patient's left mouth corner was mildly lower. Taxia was mildly hypermetric in the right upper extremity. In the sedentary position, there was instability drawn to the back, standing positions II and III were appreciably unstable. Gait was basophobic and unstable with 2 forearm crutches.

Clinically this was a central vestibular syndrome with suspect of mild ischaemic stroke in the vertebrobasilar circulation. Brain CT revealed no focal changes (Figure 1). Ultrasonography detected insignificant atherosclerotic changes in the carotid artery bifurcations and hypoplasia of the distal part of the right vertebral artery. Baseline laboratory findings were satisfactory. Electromyography was performed during hospitalisation, detecting rather severe, mainly axonal sensory-motor polyneuropathy of the lower extremities. Neurological problems (vertigo and slight dysarthria) normalized within 24 hours and patient did not receive any further medication. One explanation of this transient ischemic attack in vertebrobasilar region was microembolization while reducing the effectiveness of anticoagulant therapy in atrial fibrillation.

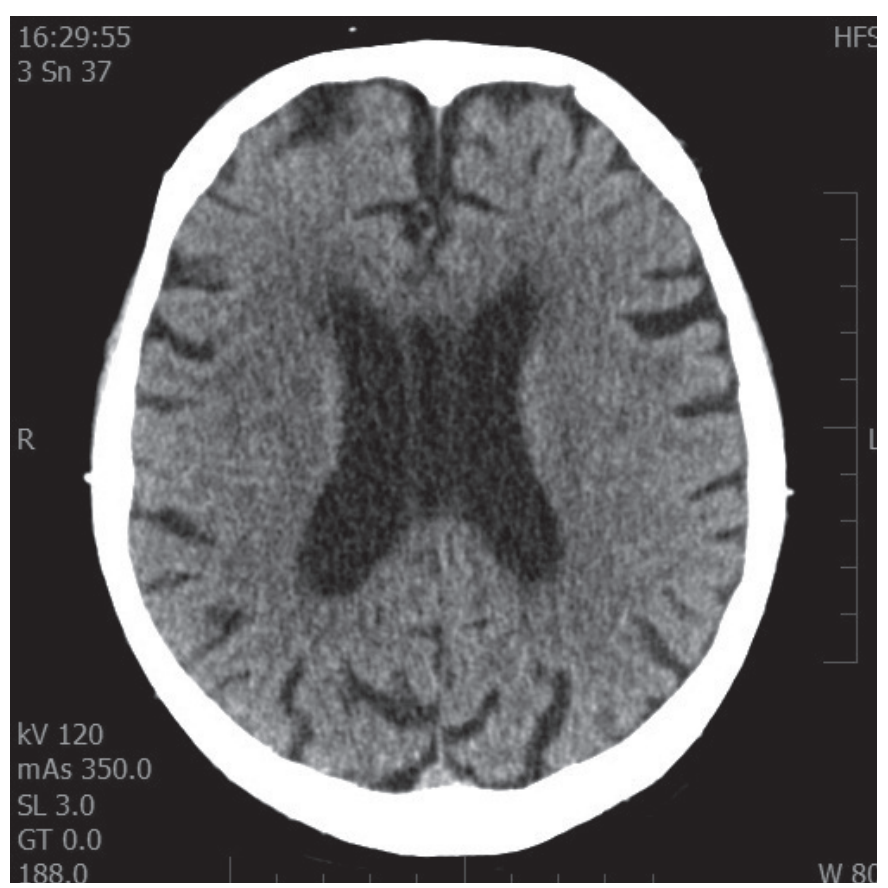

Fig. 1 Brain CT with very small isolated older hypodense foci in the bilateral periventricular area.

The patient had a low-efficient INR level on admission (see Table 2). He uses warfarin anticoagulation, dose $5 \mathrm{mg} /$ day, with good long-term stability. He brought recently measured INR records with him, all of which were in the effective range of 2-3. This way he refuted the suspected non-compliance. No dietary error could be detected either. The only major recent change was the use of the antifungal agent terbinafine against onychomycosis (about 14 days before).

After consulting the attending physician, terbinafine was discontinued. The current warfarin dosage was preserved and LMWH at an anticoagulant dose was initiated. Repeated INR measurement was recommended. 
The patient reported he was a non-smoker and never had used any food supplements with St. John's wort, tea or other food supplements.

The next INR measurement showed additional INR level decrease, despite the discontinuation of terbinafine and continued warfarin dose, which had provided a stable long-term condition (see Table 2).

Tab. 2 Patient medication and INR values in temporal sequence.

\begin{tabular}{|l|c|l|l|c|}
\hline & $\begin{array}{c}\text { Day 1 } \\
\text { (on admission) }\end{array}$ & \multicolumn{1}{|c|}{ Day 3 } & \multicolumn{1}{|c|}{ Day 4 } & $\begin{array}{c}\text { Day 7 } \\
\text { (discharge) }\end{array}$ \\
\hline Warfarin & $5 \mathrm{mg}$ & $5 \mathrm{mg}$ & $7.5 \mathrm{mg}$ & $7.5 \mathrm{mg}$ \\
\hline Terbinafine & $250 \mathrm{mg}$ & 0 & 0 & 0 \\
\hline Nadroparin & 0 & $0,6 \mathrm{ml}$ bid & $0,6 \mathrm{ml}$ bid & 0 \\
\hline INR & 1.91 & 1.53 & 1.48 & 2.4 \\
\hline
\end{tabular}

After consulting the attending physician, the warfarin dose was increased to $7.5 \mathrm{mg} /$ day and additional INR monitoring was recommended. Only the last INR lay in the therapeutic range. $\mathrm{LMWH}$ was discontinued and the patient was discharged from the hospital with a warfarin dose of $7.5 \mathrm{mg} /$ day. The attending general practitioner was instructed on the probable need to reduce the warfarin dose back to the initial $5 \mathrm{mg} /$ day after the liver enzyme induction effect completely disappears. In future, we recommend switching warfarin to some of direct anticoagulants.

\section{DISCUSSION}

The patient with paroxysmal atrial fibrillation using warfarin had an ineffective INR level caused by drug interaction with terbinafine (medication for onychomycosis); however, several medication problems were found in the patient during the initial medication review.

Few cases of warfarin-terbinafine interactions have been reported in literature $(4,5)$. Terbinafine is a potent CYP2D6 inhibitor metabolising several other drugs from among the patient's medication - propafenone, nebivolol, tramadol. Warfarin exploits three most important metabolic pathways, CYP1A2, CYP3A4 and CYP2C9. A drug interaction could lead to an increased effect of the above drugs. Enzyme induction with CYP3A4, CYP2C9, CYP1A2 inducers may have occurred. The time sequence after eliminating all the possible causes pointed to terbinafine. The induction of liver enzymes lasts 10-14 days, which matches the introduction of the antifungal agent. Furthermore, it is well-known that this induction persists after drug discontinuation, as is clear from the measured INR values.

Warfarin acts as an antagonist, antivitamin K. It prevents the synthesis of coagulation factors in the liver. The efficacy and safety of warfarin therapy is closely related to its biotransformation and high interaction potential. Warfarin is among drugs with a narrow therapeutic index and a poorly predictable effect due to genetic polymorphism - CYP2C9 oxidase, which provides its biotransformation. The effect of warfarin should therefore be monitored based on periodic INR measurement. Its mechanism of action is based on the effect on vitamin $\mathrm{K}$ epoxide reductase and vitamin $\mathrm{K}$ reductase, two key enzymes of vitamin $\mathrm{K}$ metabolism. Warfarin is a racemic mixture of two enantiomers $(\mathrm{R}, \mathrm{S})$ with different pharmacokinetic and pharmacodynamic properties. The most important drug interactions take place at the level of hepatic metabolism, i.e., inhibition or induction. Warfarin is metabolised via three forms of cytochrome P-450: R-form CYP1A2 and CYP3A4, S-form: CYP2C9 (6). Drugs that inhibit these enzymes can cause reduced warfarin conversion, with consequent accumulation of the active substance in plasma and subsequently a higher anticoagulation effect. Conversely, a number of active substances induce the production of cytochromes $\mathrm{P} 450$ involved in warfarin metabolism, with a consequent decrease in metabolised isomer levels and possible failure of the anticoagulation therapy.

Terbinafine is a fungicidal allylamine antifungal agent. It inhibits squalene epoxidase, the enzyme responsible for ergosterol synthesis, which leads to impaired cell wall function. It is not used in systemic mycoses because its bonding to plasma proteins is very strong and effective concentrations are only attained in the skin and its adnexes. Thus, terbinafine is normally primarily used against onychomycoses. It is well absorbed on oral administration, binds strongly to plasma proteins, and is biotransformed in the liver by CYP2C8, CYP2C9, CYP2C19 and CYP3A4 oxidases to inactive metabolites. It is a potent inhibitor of CYP2D6, its half-life is 22-26 hours and even as long as 200-400 hours in skin adnexa. However, terbinafine may be considered as a weak liver enzyme inducer as well but this interaction will only be clinically apparent in susceptible patients (the role of genetic polymorphism) (7).

\section{CONCLUSION}

Combined therapy with warfarin and terbinafine is actually rarely prescribed but, if used, their interaction can have serious consequences in many clinical situations for which anticoagulation therapy with warfarin is indicated.

\section{ACKNOWLEDGEMENTS}

Supported by the Research projects of Charles University PROGRES Q 35 and UNCE/MED/002.

REFERENCES

1. Bjorck S, Palaszewski B, Friberg L, Bergfeldt L. Atrial fibrillation, stroke risk, and warfarin therapy revisited: a population-based study. Stroke 2013; 44: 3103-8.

2. Hart RG, Pearce LA, Aguilar MI. Meta-analysis: antithrombotic therapy to prevent stroke in patients who have nonvalvular atrial fibrillation. Ann Intern Med 2007; 146: 857-67.

3. Heidbuchel H, Verhamme P, Alings M, et al. Updated European Heart Rhythm Association practical guide on the use of non-vi- 
tamin-K antagonist anticoagulants in patients with non-valvular atrial fibrillation: executive summary. Eur Heart J 2017; 38: 2137-49.

4. Gantmacher J, Mills-Bomford J, Williams T. Interaction between warfarin and oral terbinafine. Manufacturer does not agree that interaction was with terbinafine. BMJ 1998; 317(7152): 205; author reply 205.
5. Warwick JA, Corrall RJ. Serious interaction between warfarin and oral terbinafine. BMJ 1998; 316: 440.

6. Kaminski LS, Zhang Z-Y. Human P450 metabolisms of warfarin. Pharmacol Ther 1997; 73(1):67-74.

7. Venkatakrishnan K, von Moltke LL, Greenblatt DJ. Effects of the antifungal agents on oxidative drug metabolism: clinical relevance. Clin Pharmacokinet 2000; 38(2): 111-80. 